\title{
Midazolam sedates agitated patients more rapidly than haloperidol-promethazine
}

TREC Collaborative Group. Rapid tranquillisation for agitated patients in emergency psychiatric rooms: a randomised trial of midazolam versus haloperidol plus promethazine. BMJ 2003;327:708-13.

$Q$ Is midazolam or haloperidol plus promethazine more effective for rapid sedation of agitated or aggressive people with mental illness?

\section{METHODS}

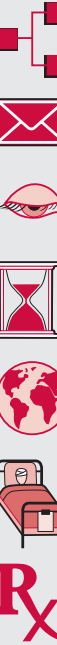

Design: Randomised controlled trial.

Allocation: Concealed.

Blinding: Treatment was open, but assessors were blinded to intervention.

Follow up period: Twenty minutes for primary outcome.

Setting: Three psychiatric emergency rooms, Rio de Janeiro, Brazil.

Patients: 301 people needing sedation for agitation or aggressive behaviour.

R Intervention: Intramuscular injection of midazolam $15 \mathrm{mg}$ or $7.5 \mathrm{mg}$ ) or haloperidol plus promethazine $15 \mathrm{mg}$ or $10 \mathrm{mg}$ haloperidol plus $50 \mathrm{mg}$ promethazine).

- Outcomes: Participant asleep or tranquillised at 20 minutes;

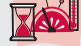
adverse effects.

$\square$ Patient follow up: $99 \%$.

\section{MAIN RESULTS}

Midazolam rendered significantly more participants asleep or tranquil in 20 minutes than haloperidol plus promethazine (AR $89 \%$ with midazolam $v 67 \%$ with haloperidol+promethazine; RR 1.32, 95\% CI 1.16 to 1.49 ; NNT 5, 95\% CI 3 to 8 ; analysis by intention to treat). Adverse effects: one person receiving midazolam had transient respiratory depression; one person receiving haloperidol plus promethazine had a grande mal seizure.

For correspondence: Dr G Huf, Universidade Federal do Rio de Janeiro, Núcleo de Estudos de Saúde Coletiva, Av. Brigadeiro Trompowsky $\mathrm{s} / \mathrm{n}$, Edifício Hospital Universitário 5 andar, ala sul - Itha do Fundão, Rio de Janeiro, Brazil, 21941-590, Caixa Postal 68037; gisele@ensp.fiocruz.br Sources of funding: no participating centre directly received funds for involvement in TREC.

\section{CONCLUSIONS}

Midazolam sedated participants more rapidly than haloperidol plus promethazine. Its use could decrease the time during which care workers are exposed to aggressive or agitated behaviour.

\section{Commentary}

The TREC trial is one of the first larger scale pragmatic randomised controlled trials in mental health research ${ }^{1}$ and should be viewed as a significant milestone. As a pragmatic trial it was designed and conducted in a "real world" clinical setting with the aims of enhancing generalisability and decreasing study attrition. ${ }^{2}$ TREC is pragmatic in several ways; it avoids complex protocols that would disrupt routine care, it has simple eligibility criteria and it simplifies data collection. It was clearly successful in minimising study attrition; $99 \%$ completion was attained, a rate unheard of in most trials. ${ }^{3}$ The conduct of this trial in a middle income country should also enhance worldwide generalisability.

The authors ensured a clinically relevant primary outcome (20 minute sedation) by polling participating front line clinicians. The interrater reliability of this newly defined outcome was found to be high $(\mathrm{kappa}=0.83$ ). The efficacy finding of the study favouring midazolam over haloperidol+promethazine is likely be clinically relevant in jurisdictions where these agents are typically used. This study may not have impact in jurisdictions where other agents such as intramuscular lorazepam, loxapine, perphenazine, olanzapine, or risperidone are used. Other simple pragmatic trials should be conducted to compare such agents.

An unresolved issue from this trial is that of comparative safety. Midazolam is associated with respiratory depression ${ }^{5}$ which is why it is typically used within surgical and intensive care settings. ${ }^{5} 6$ The authors may overstate their case when they assert there is sufficient evidence for midazolam to be used safely in emergency settings (even with the availability of flumazenil) as the trial is under powered to examine this outcome. A larger pragmatic trial or an examination of administrative linked databases, including pharmaceutical data and serious adverse events in emergency settings, is needed to clarify this safety issue.

Paul S Waraich, MHSc MD FRCP(C) Research Associate, MHECCU, Department of Psychiatry, UBC, Vancouver, Canada

1 Hotopf $M$, Churchill R, Lewis G. Pragmatic randomised controlled trials in psychiatry. Br J Psychiatry 1999:175:217-23.

2 Roland M Torgerson DJ. What are pragmatic trials? BMJ 1998:316.285.

3 Wahlbeck K, Tuunainen A, Ahokas A, et al. Dropout rates in randomised antipsychotic drug trials. Psychopharmacology (Berl) 2001;155:230-3.

4 Hartke RH Jr, Gonzalez-Rothi RJ, Abbey NC. Midazolam-associated alterations in cardiorespiratory function during colonoscopy. Gastrointest Endosc 1989:35:232-8.

5 Shelly MP, Sultan MA, Bodenham A, et al. Midazolam infusions in critically ill patients. Eur J Anaesthesiol 1991;8:21-7. 